\title{
Cost-effectiveness of interferon- $\gamma$ release assay testing for the treatment of latent
} tuberculosis

\author{
R. Diel*, P. Wrighton-Smith ${ }^{\#}$ and J-P. Zellweger ${ }^{*}$
}

ABSTRACT: The aim of the present study was to assess the cost-effectiveness of the new T-SPOT.TB assay versus the tuberculin skin test (TST) for screening contacts for latent tuberculosis (TB) infection in Switzerland.

Health and economic outcomes of isoniazid treatment of 20- and 40-yr-old close contacts were compared in a Markov model over a 20 -yr period following screening with TST only (at three cutoff values) and T-SPOT.TB alone or in combination with the TST.

T-SPOT.TB-based treatment was cost-effective at $€ 11,621$ and $€ 23,692$ per life-year-gained (LYG) in the younger and older age group, respectively. No TST-based programmes were costeffective, except at a 15-mm cut-off in the younger group only, where the cost-effectiveness $\left(€ 26,451 \cdot \mathrm{LYG}^{-1}\right)$ fell just below the willingness-to-pay threshold. Combination of the TST with T-SPOT.TB slightly reduced the total cost compared with the T-SPOT.TB alone by 4.4 and $5.0 \%$ in the younger and older groups respectively. The number of contacts treated to avoid one case of TB decreased from 50 (95\% confidence interval 32-106) with the TST (10-mm cut-off) to $18(95 \% \mathrm{Cl}$ 11-43) if T-SPOT.TB was used.

Using T-SPOT.TB alone or in combination with the tuberculin skin test for screening of close contacts before latent tuberculosis infection treatment is highly cost-effective in reducing the disease burden of tuberculosis.

KEYWORDS: Cost-effectiveness, interferon- $\gamma$ release assay, latent tuberculosis infection, latent tuberculosis infection treatment, tuberculosis

creening the contacts of patients with tuberculosis (TB) is recommended as a strategy to detect infected persons who may develop the disease at a later time. It has been demonstrated that preventive treatment, mainly with isoniazid, decreases the number of future cases of TB. This strategy is therefore recommended in countries with a low incidence rate of $\mathrm{TB}$, in order to further decrease the burden of disease [1]. The effectiveness and cost-effectiveness of these programmes are strongly affected by the accuracy of identifying truly infected individuals who have a risk of developing future disease. Owing to the limited sensitivity and specificity of the tuberculin skin test (TST), it follows that the current cost-effectiveness of screening may be improved if more accurate tools are used for screening for latent tuberculosis infection (LTBI).

Numerous studies screening recent contacts of infectious TB patients for LTBI using the new highly specific interferon- $\gamma$ release assays (IGRA) have recently been published [2-7], but no study has produced cost-effectiveness data. In two papers $[8,9]$ the way in which IGRA can be used for cost-saving in initial screening has been discussed. However, the long-term economic consequences and healthcare outcomes of this new approach for detecting Mycobacterium tuberculosis infection were not examined in the context of subsequent treatment of LTBI in comparison with existing programmes based upon the TST.

As intervention options in all therapeutic areas grow, government and third-party payers, which are under increasing budgetary constraints, are seeking ways in which they can allocate resources in order to achieve maximum benefits for healthcare. Therefore, the present authors conducted a cost-effectiveness analysis of several different LTBI screening strategies followed by isoniazid treatment under a range of different conditions. In Switzerland, currently published recommendations [10] suggest the implementation of IGRA because of their enhanced specificity
AFFILIATIONS

*School of Public Health, University of Düsseldorf, Germany.

\#0xford Immunotec, Oxford, UK.

"University Medical Policlinic,

Lausanne, Switzerland.

CORRESPONDENCE

R. Diel

School of Public Health

c/o Institute for Medical Sociology

Heinrich Heine University

Post box 101007

D-40001 Düsseldorf

Germany

Fax: 4940427907185

E-mail: Roland.Diel@

uni-duesseldorf.de

Received:

November 072006

Accepted:

April 192007

STATEMENT OF INTEREST

A statement of interest for P. WrightonSmith can be found at

www.erj.ersjournals.com/misc/

statements.shtml 
over the TST as confirmatory tests for TST-positive contacts, in order to minimise the number of subjects treated unnecessarily for LTBI. Therefore, the study was based on current Swiss epidemiological and cost data. As these guidelines (and the previous cost-saving analyses) only consider the increased specificity of the IGRA, they do not take into account any healthcare gains resulting from any increased sensitivity of one or both of the IGRA over the TST. Both because the current authors were able to use data directly from routine clinical use of the test in Switzerland, and because the available evidence suggests that it is the most sensitive of the two IGRA [11, 12], it was decided to model the cost-effectiveness of the T-SPOT.TB test.

\section{MATERIALS AND METHODS \\ Screening strategies}

Five strategies were considered. Strategies 1-3 reflect current practice, in which the TST is used as the only tool to diagnose LTBI using the Swiss-standard induration cut-off ( $\geqslant 10 \mathrm{~mm})$, but also two further commonly used cut-offs ( $\geqslant 5$ and $\geqslant 15 \mathrm{~mm}$ ). Strategy 4 calculated the consequences of using the T-SPOT.TB test alone (i.e. a complete replacement for the TST), and strategy 5 calculated the cost-effectiveness following the recommendation as described above, i.e. using the TST with a cut-off of $\geqslant 10 \mathrm{~mm}$ for the initial screening of patients, followed by a T-SPOT.TB test in all TST-positive individuals before treatment.

\section{Decision analysis model}

Using the decision analysis software program TreeAge Pro 2006 Healthcare Module, Release 0.2 (TreeAge Software Inc., Williamstown, MA, USA) a Markov model was developed tracing the contacts' economic and healthcare outcomes resulting from the test results of each strategy and two different age adult close contact groups (a young group, with a mean age of $20 \mathrm{yrs}$, and a middle-aged group of mean age $40 \mathrm{yrs}$ ). A hypothetical cohort of 1,000 individuals was used for the analysis, taken by normalising the actual data (table 1).

The resulting decision tree (showing in this case the T-SPOT.TB screening strategy) is presented in figure 1 . The tree is entered from the left, where the whole cohort begins at time zero as contacts. If the test results are negative, these persons are considered not to be infected, but in reality a certain proportion of them may be "false negative", depending upon the assumed sensitivity of the test. Contacts whose results are positive go on to undergo chest radiography to exclude active TB, and are then assumed to have LTBI and offered isoniazid treatment. It is assumed that a 9-month course of isoniazid provides protection with an efficacy of $80 \%$ (see below) for 20 yrs [13] and that no reinfections with $M$. tuberculosis will occur. For modelling purposes two scenarios were followed prospectively; one where all contacts accept isoniazid and one where none do.

The Markov model simulates the natural history of TB disease, with people passing through a number of health states, defined to capture important clinical outcomes, each of which is associated with specific costs and rewards (in this case survival time). Consistent with the assumed duration of isoniazid protection, the present study used a Markov model incorporating 20 equal annual iterations over a 20 -yr period.

The following five mutually exclusive health states included in the present model describe the various possible states of close contacts after they have been infected with M. tuberculosis: 1) asymptomatic LTBI; 2) active illness due to reactivation, to which some of these LTBI cases progress owing to reactivation (with a transition probability denoted tpReact); 3) TB disease, leading to death due to the disease itself (including consequent conditions; transition probability denoted tpDcm); or in contrast 4) survival after recovery without sequelae (1-tpDcm); or 5) death due to "normal" all-cause mortality, excluding TB disease, represented by age-dependent life expectancy, with a probability tpDn that is taken to affect all patients equally (whether in the LTBI or survival state).

\section{Probabilities}

Probabilities of transitions between states representing the best available data are shown in table 2 .

\section{Risk of death}

The background likelihood of death unrelated to TB disease (tpDn) occurring in the general population is time-dependent, increasing with age. Data were based on the current Swiss life tables [19] and weighted according to the different life expectancies of males and females.

In Switzerland in 2004, a total of 24 out of 658 persons suffering from TB died from the disease [20], resulting in a baseline rate of $3.7 \%(\mathrm{tpDcm})$.

\section{Isoniazid efficacy}

As it is described in detail elsewhere [13], it was assumed that a 9-month isoniazid course would have an $80 \%$ efficacy rate (effect) in preventing progression to active TB disease.

\begin{tabular}{|c|c|c|c|c|c|c|c|}
\hline \multirow[t]{2}{*}{ T-SPOT.TB result } & \multicolumn{2}{|c|}{ TST $\geqslant 5 \mathrm{~mm}$} & \multicolumn{2}{|c|}{$\mathrm{TST} \geqslant 10 \mathrm{~mm}$} & \multicolumn{2}{|c|}{$\mathrm{TST} \geqslant 15 \mathrm{~mm}$} & \multirow[t]{2}{*}{ Total } \\
\hline & Negative & Positive & Negative & Positive & Negative & Positive & \\
\hline Negative & $49(184)$ & 144 (539) & 65 (243) & $128(479)$ & $130(487)$ & $63(236)$ & 193 (723) \\
\hline
\end{tabular}




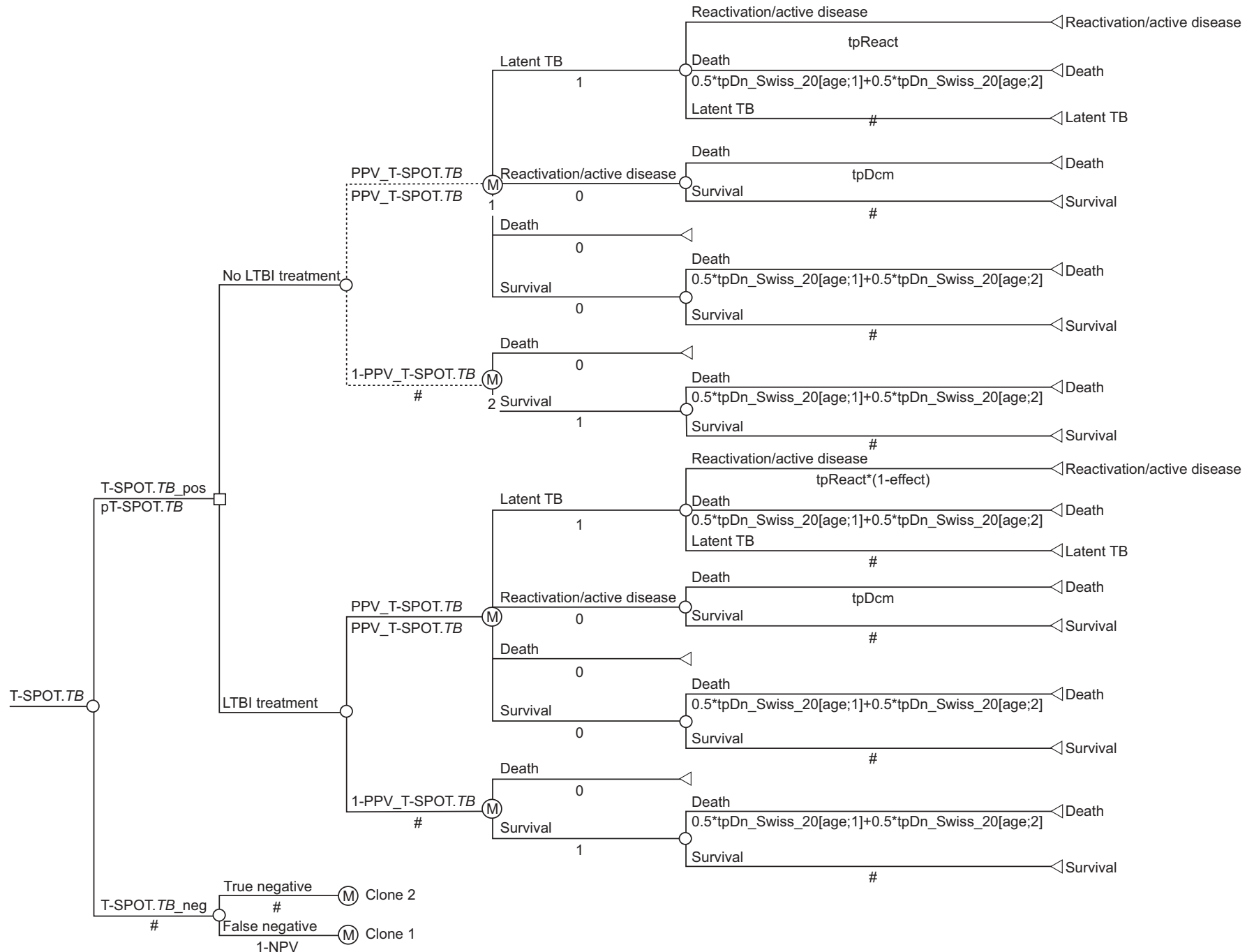

FIGURE 1. Markov model for predicting the total costs and the occurrence of tuberculosis (TB) due to latent TB infection (LTBI) by screening a cohort of 20-yr-old close contacts and treating the test-positives with isoniazid using the T-SPOT.TB strategy. A decision node $(\square)$ is the decision after a positive T-SPOT.TB test whether to accept isoniazid. Branches from a change node $(O)$ represent the possible outcomes of an event; branches from a markov node $(M)$ represent the possible different health states. A terminal node $(\triangleleft)$ represents a state from which an individual will jump to the next cycle. Only the "death" state is an absorbing one and cannot be departed from. .......... the following subtree is cloned, i.e. a copy is attached to a node in another branch of the tree. The cloned subtrees, denoted clone 1 and clone 2 , are attached to the false negative and the true negative node, respectively. T-Spot.TB_pos: positive result of T-SPOT.TB test; PT-Spot.TB: probability of T-SPOT.TB being positive; T-Spot.TB neg: negative result of T-SPOT.TB test; NPV: negative predictive value; PPV: positive predictive value; tpReact: transition probability for a progression to active TB; tpDn_20: probability of death due to causes other than TB among contacts with an initial age of 20; tpDcm: probability of death due to TB; effect: \% efficacy of isoniazid to prevent progression to manifest TB. ${ }^{*}$ : complementary probability (all probabilities of chance node's branches to sum to 1).

Risk of reactivation

The risk of TB reactivation (tpReact) depends largely on two risk factors: the age of the infected person and the size of induration produced by a TST. The individual risk of close contacts as recent converters were derived using the metaanalysis of HORSBURGH [21] for the two age-groups separated by the three induration diameters 5,10 and $15 \mathrm{~mm}$. Owing to the long period of isoniazid protection (20 yrs), these values were translated into a fixed transition per year (cycle) and did not take into account the increased risk of reactivation within the first 2 yrs following infection. Although it might have been expected that the T-SPOT.TB test would have a higher positive predictive value (PPV) than the TST for the eventual development of TB disease (see Discussion section), the conservative assumption was made that the risk of $\mathrm{TB}$ reactivation following a positive T-SPOT.TB test was equal to that of the TST at a 10-mm cut-off (the current Swiss standard) as a baseline value and it was increased to that of the TST at $15 \mathrm{~mm}$ in the sensitivity analysis (the "high reactivity rate").

\section{Data inputs for T-SPOT.TB and TST}

Method-related data for this analysis were taken from a recent side-by-side comparison of the TST with T-SPOT.TB among 267 adult close contacts under routine programme conditions at Lausanne University Medical Polyclinic (Lausanne, Switzerland) between January 2004 and December 2005. This population contained a high proportion of Bacille CalmetGuérin (BCG) vaccinees [9]. The TST was applied by the 


\section{TABLE 2 Base-case estimates used in cost-effectiveness analysis}

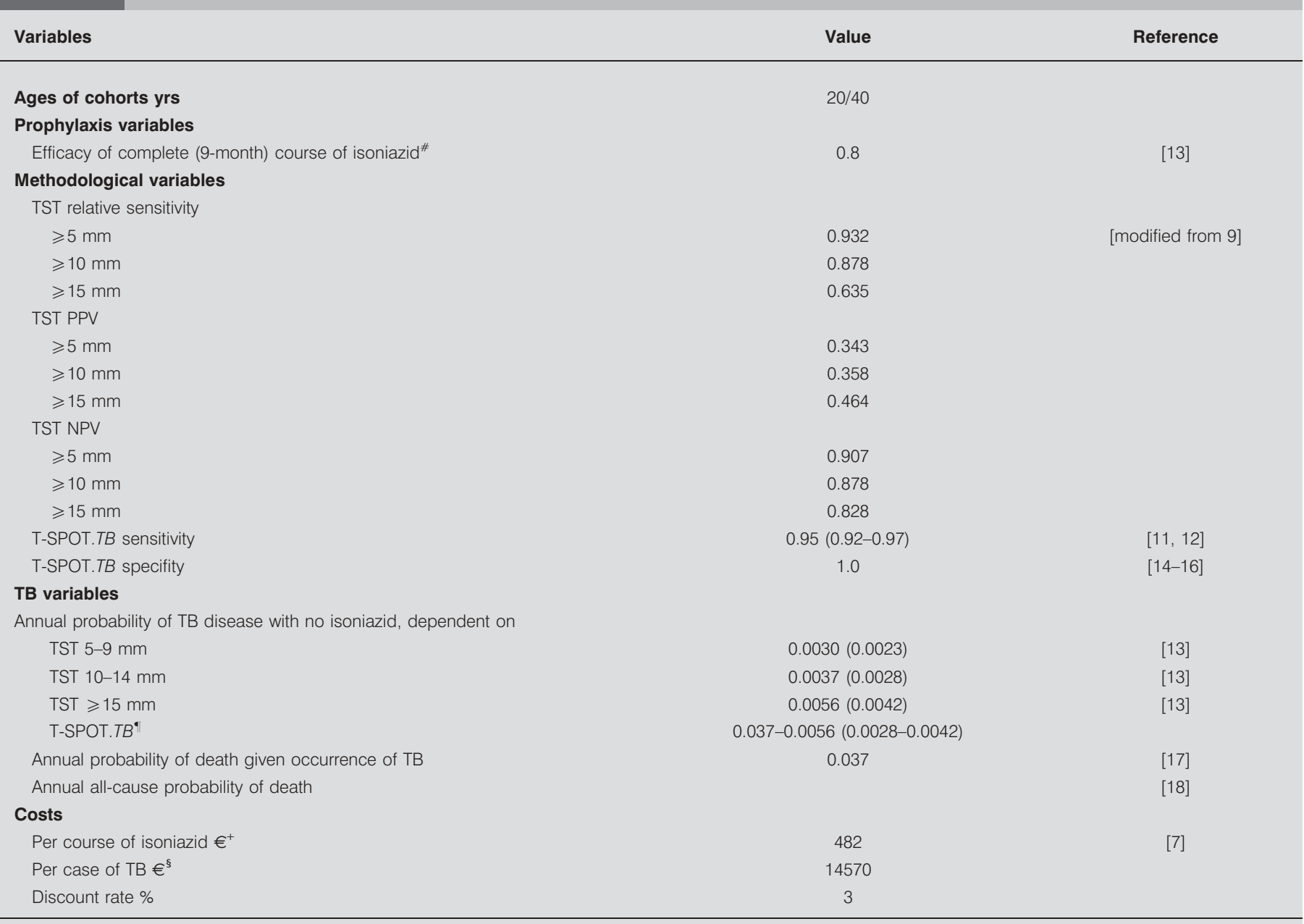

Data are presented as value, value (range) or value for 16-35-yr-old converters (value for 36-55-yr-old converters), unless otherwise stated. TST: tuberculin skin test; PPV: positive predictive value; NPV: negative predictive value; TB: tuberculosis. ${ }^{*}$ : annual reduction in probability of developing TB; $^{~ ": ~ b a s e d ~ o n ~ a n ~ a s s u m p t i o n ; ~}{ }^{+}$: daily for 9 months; s. model estimation.

Mantoux method, using 2 TU of RT23 PPD according to the Swiss National Guidelines [22]; results were read at $72 \mathrm{~h}$ and considered positive if induration was $\geqslant 10 \mathrm{~mm}$; the individuals in question were offered preventive treatment with isoniazid for 9 months. For the T-SPOT.TB, a 10-mL blood sample was taken and analysed in a local laboratory (BBR-LTC laboratories, Lausanne, Switzerland); the cut-off for the assay was 6 spots, according to the manufacturer's instructions (Oxford Immunotec, Abingdon, UK).

The raw data on the results of both tests, separated by three different TST cut-offs in order to investigate the concordance between the T-SPOT.TB and various TST induration diameters are shown in table 1 . These values were then normalised to a cohort size of 1,000 subjects (table 1 ) and used to calculate inputs for the modelling.

The sensitivity and specificity for T-SPOT.TB were taken from the published literature. In culture-confirmed active TB patients, the sensitivity of T-SPOT.TB in largely immunocompetent populations has been reported at 95.4-97.2\% [11, 17, 18] Higher sensitivity has also been consistently observed for the T-SPOT.TB assay over the TST in LTBI $[2-3,5-7]$ and thus a conservative baseline figure of $95 \%$ sensitivity for LTBI was taken for the present analysis. The specificity of the T-SPOT.TB assay in low-risk healthy controls approaches 100\% [14-16].

Assuming a $95 \%$ sensitivity and $100 \%$ specificity for T-SPOT.TB, it follows that there are no false-positive T-SPOT.TB results, but that $5 \%$ of truly infected people are recorded falsely as negative. Hence, if 277 subjects are recorded as positive by T-SPOT.TB, then $291.6(277 / 0.95)$ subjects in the starting cohort for the analysis must have been truly infected. This in turn allows the model to calculate the resulting costs and sequelae from those persons in whom true LTBI is missed. As can be seen from figure 1, the model is constructed so that the probability of a true negative result is taken directly from the negative predictive value (NPV) of the test (i.e. true negative results/total negative results). For the T-SPOT.TB test, the NPV can be calculated as (723-14.6)/723 $=98 \%$. Likewise, the 
probability of a true positive result was taken from the PPV of the test, which for T-SPOT.TB, as it is assumed to have $100 \%$ specificity (i.e. no false-positives), is $100 \%$.

Sensitivity and specificity (and hence the NPV and PPV) were calculated for the TST relative to T-SPOT.TB based upon the recorded comparative data between the tests. Clearly, given the absence of a gold-standard test for LTBI, there is no way of knowing which test is correct where the results are discordant. Two assumptions, as described below, were therefore made. As T-SPOT.TB is assumed to have no false-positive results (100\% specificity) all TST-negative, T-SPOT.TB-positive responses were approximated as false-negative TST results and the sensitivity of the TST was calculated accordingly. There were 5, 9 and 27 individuals who were T-SPOT.TB-positive, but TST-negative at cut-off values of 5, 10 and $15 \mathrm{~mm}$, respectively (table 1), indicating that the TST test has a sensitivity, relative to the T-SPOT.TB, of 93.2, 87.8 and only $63.5 \%$, respectively. The corresponding NPVs for the TST are $90.7 \%$ (49 out of 54 ) at a cut-off of $5 \mathrm{~mm}, 87.8 \%$ (65 out of 74 ) at a cut-off of $10 \mathrm{~mm}$ and $82.8 \%$ (130 out of 157) at a cut-off of $15 \mathrm{~mm}$. To calculate the PPV for the TST, an assumption must be made of how many of the TST-positive subjects are truly infected. It cannot automatically be inferred that only those with also a positive T-SPOT.TB result are infected as T-SPOT.TB is assumed to only have $95 \%$ sensitivity and thus will miss some individuals who may be picked up by the TST. Despite the evidence that T-SPOT.TB is uniformly more sensitive than the TST $[2,3,5-7]$, the present authors made the conservative assumption that all T-SPOT.TB false-negatives would be picked up by the TST. From table 1, the number of TSPOT.TB false negatives was calculated as $74 / 0.95=77.9$; $77.9-74=3.89$; i.e. four cases rounded; it was assumed these four were picked up by the TST. Consequently the PPV of the TST was calculated as $34.3 \%(73(69+4) / 213)$ at $5 \mathrm{~mm}, 35.8 \%$ $(69(65+4) / 193)$ at $10 \mathrm{~mm}$ and $46.4 \%(51(47+4) / 110)$ at $15 \mathrm{~mm}$.

\section{Estimation of costs}

Costs were expressed in 2004 Swiss francs (CHF) and converted to euros where appropriate (at a rate of CHF $1=€ 0.645$ ).

\section{Costs of LTBI screening and treatment}

The costs of LTBI testing and treatment were recently published in a cost minimisation study from the Swiss healthcare perspective [9]. Asymptomatic infection is assumed to produce no cost (except the cost of testing, which would have been incurred irrespective of infection). The costs of testing comprised the labour cost for the staff performing the TST or drawing blood, as well as the material cost of the vial and associated consumables for each TST at CHF 35 (€23). As reimbursement has not yet been formalised for the T-SPOT.TB test in Switzerland, an estimate for the total cost of the screening kit, reagents and laboratory fees was taken as CHF $200(€ 129)$ for each T-SPOT.TB test as previously described [9].

Treatment costs include initial chest radiography to rule out active TB prior to treatment, the costs of 9 months' isoniazid treatment and the costs of visits to the clinician and liverfunction tests during the treatment period (table 3). Sideeffects from isoniazid treatment were ignored, and all patients for whom preventative therapy was indicated were assumed to complete the full course of therapy.

\section{Cost of illness}

The overall cost of TB disease per person was calculated from the Swiss social perspective. Thus, both direct costs for in- and outpatients (comprising also the contact-tracing induced by infectious pulmonary source cases) as the sum of the average costs for each clinical outcome weighted by the probability of occurrence of that outcome and productivity losses due to illness were included in cost estimates. The baseline cost estimates are shown in table 4.

\section{Epidemiological data}

Out of the 2,485 TB cases reported in Switzerland between 2001 and 2004, 1,861 ( 75\%) were pulmonary. Out of these, 524 (28\%) were sputum-smear-positive, 954 (51\%) were sputumnegative but culture-confirmed, and only 383 (21\%) had been clinically diagnosed without bacteriological confirmation [23].

Although hospitalisation is recommended for smear-positive cases according to the current guidelines [24], only $\sim 84 \%$ of those had been treated as in-patients in 2004, with an average stay in hospital of $\sim 25$ days; $27 \%$ of the remaining pulmonary TB cases had been hospitalised (average stay 21 days) and 16\% of the nonpulmonary TB cases (average stay 10 days), for whom hospitalisation is optional (if there is comorbidity; individual assessment by the Swiss Federal Office of Public Health, not including long-term in-patients).

\section{Direct costs}

The diagnostic and treatment costs of smear-positive TB patients in 2004 amounted to CHF 39,659 per patient. Examination usually integrates three chest radiographical scans (at diagnosis, after 2 months and at the end of treatment) and four smear examinations (two at the start, one after 2 months, and one before the end of treatment, with strain identification and a drug sensitivity test performed on the first sample). Treatment is usually 2 months' isoniazid + rifampin + pyrazimade + ethambutol $/ 4$ months' isoniazid + rifampin, according to the World Health Organization recommendations.

For sputum-negative patients the costs amount to CHF 33,117 (only two sputum examinations and cultures at the start and only 21 days of hospital stay); nonpulmonary cases cost CHF 16,678 (10 days in hospital, only one chest radiographical examination) to treat. Before diagnosis at least two clinical visits (CHF 58 each) are necessary.

Treatment is self-administered in the majority of cases, supervised by a member or representative of the health system observing each medication intake by the TB patient (directly observed therapy (DOT)) in cases with a risk of nonadherence (drug addicts, alcoholics, psychiatric cases, elderly persons with disorientation, immigrants not yet socially integrated, relapses, multiple drug resistant (MDR)-TB) to prevent the development of drug resistance. In 2004, 68 (11\%) out of the 606 Swiss TB cases were started on DOT (the proportion varied according to location, being higher in large cities than in the countryside) and remained on it for an average of 4 months. Of these cases, $\sim 75 \%$ were charged CHF $10 \cdot$ day $^{-1}$ for administration under supervision at a dispensary 5-6 times a week, 


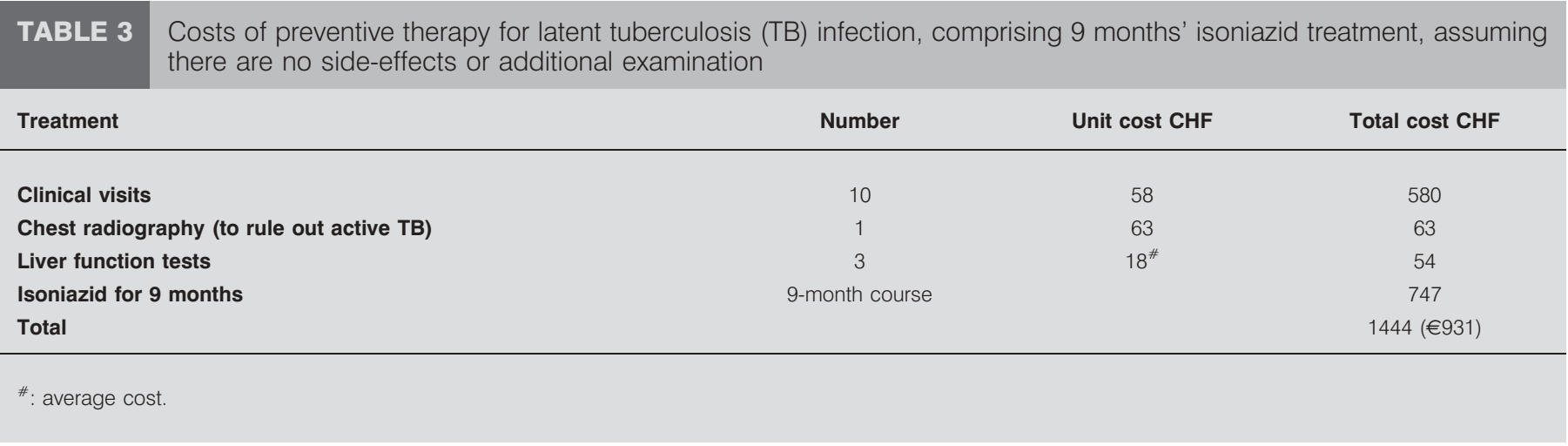

giving a monthly cost of $\sim \mathrm{CHF} 200$ (end of treatment is usually self-administered), and $\sim 25 \%$ were given their medication by a nurse (at a house visit) 5-6 times a week, at a cost of CHF $120 \cdot h^{-1}$; equivalent to $\sim \mathrm{CHF} 2,500 \cdot \mathrm{month}^{-1}$. The average cost of DOTS normalised over the entire cohorts was therefore calculated as follows: $11 \%$ of patients receiving DOTS $\times$ average duration of DOTS $\times(75 \%$ of patients under selfadministration $\times$ monthly cost of self-administration $+25 \%$ of patients given nurse medication $\times$ monthly cost of nurse medication). Inserting the relevant values, the calculation is:

$$
0.11 \times 4 \times(0.75 \times 200+0.25 \times 2500)=\mathrm{CHF} 341
$$

The costs of MDR-TB, which is rare $(2 \%$ of the cases in Switzerland), and additional costs of special examinations (computed tomography scans, biopsies) for nonpulmonary TB are not included in this listing of costs.

In Switzerland, contact-tracing is performed by order of the local public health officer, for all cases of smear-positive pulmonary TB and in some cases of smear-negative TB if there are small children or immunocompromised persons among the contacts. In 2004, 216 contact-tracings were performed for smear-positive index cases, leading to examination of a total of 3,578 individuals. Therefore, one source case with at least culture-confirmed TB will bring about the investigation of $\sim 16$ contacts; this will be organised by a nurse spending $\sim 1 \mathrm{~h}$ per contact at a charge of CHF 120 each; giving CHF 1,920 on average for every (at least culture-confirmed) TB patient.

\section{Indirect costs}

In 2004, the average sick-leave duration of TB cases (all forms) was 2 months (60 days) per case (unpublished data). In accordance with the human capital approach [25], indirect costs addressing the production loss for the economy as a whole are caused by absence from the workplace on sick leave. According to the Hanoverian Consensus [25], the productivity losses caused by sickness should be evaluated without consideration of differences in the nature of the work, or of differences in age or sex, using the average gross Swiss income for 2005. The average productivity loss is calculated as follows: productivity loss $=$ number of TB-related days of work lost $\times$ (average gross income per year $/ 365$ days) $\times$ employment rate. If the employee pay (2005 yearly average $=$ CHF 74,200 [26]) per day is multiplied by the 60 sick-leave days, this results in a total of CHF 12,197.26 as the average indirect costs per adult patient. Multiplication by the employment rate for 2004 (56.2\% [27]) then results in a cost of CHF 6,854.86.

Thus, the average weighted overall TB costs in CHF produced by a model patient may be calculated as follows: ((treatment cost

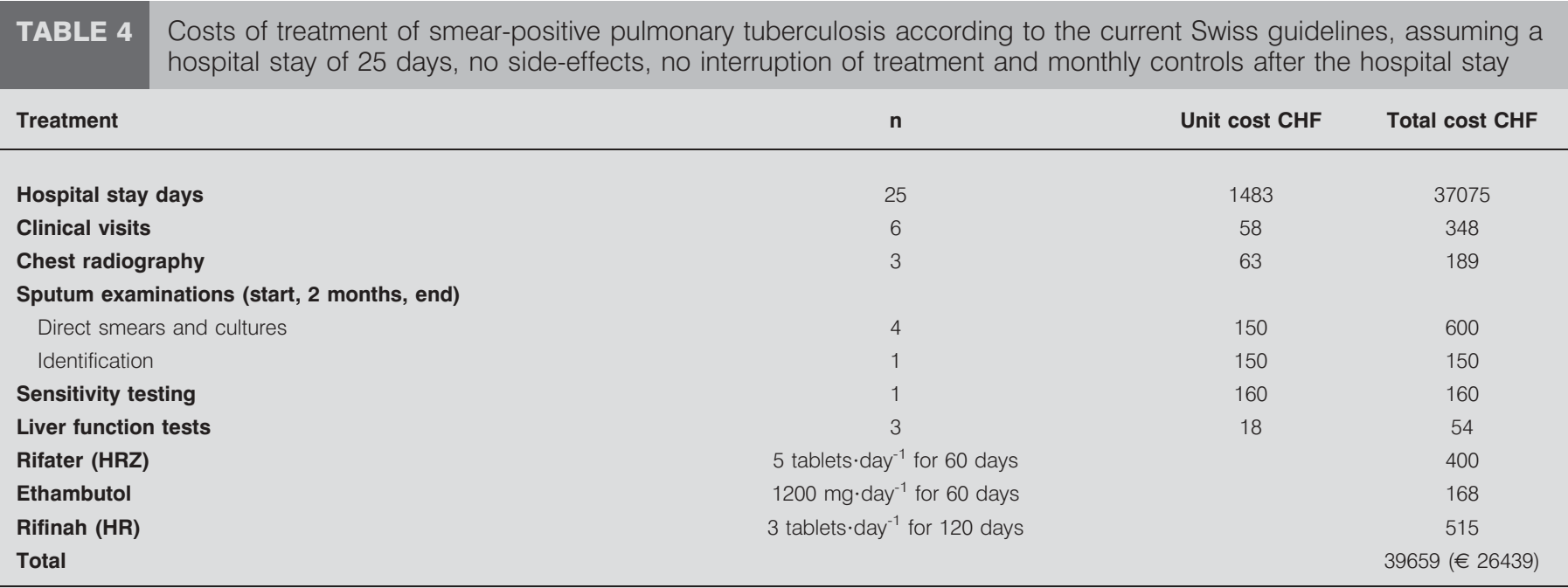

HRZ: isoniazid + rifampin + pyrazimade; HR: isoniazid + rifampin. 
of smear-positives $\times \%$ of in-patients $\times \%$ of smear-positives) + (treatment cost of smear-positives without hospitalisation $\times$ $\%$ of outpatients $\times \%$ of smear-positives) + (treatment cost of smear-negatives $\times \%$ of in-patients $\times \%$ of smear-negatives) + (treatment cost of smear negatives without hospitalisation $\times \%$ of outpatients $\times \%$ of smear-negatives) $) \times \%$ of pulmonary TB cases $+($ (treatment cost for nonpulmonary TB $\times \%$ of inpatients $)$ + (treatment cost for nonpulmonary TB without hospitalisation $\times \%$ of outpatients) $) \times \%$ of nonpulmonary TB cases + cost of visits before diagnosis + DOT cost + (cost for contact-tracing $\times$ $\%$ of pulmonary TB $\times \%$ of culture confirmed cases $)+$ indirect costs.

Inserting the corresponding values, the current authors calculated:

$((\mathrm{CHF} 39,659 \times 0.84 \times 0.28)+(\mathrm{CHF} 2,584 \times 0.16 \times 0.28)+(\mathrm{CHF}$ $33,117 \times 0.27 \times 0.72)+(\mathrm{CHF} 2,584 \times 0.73 \times 0.72)) \times 0.75+((\mathrm{CHF}$ $16,678 \times 0.16)+($ CHF $2,584 \times 0.84)) \times 0.25+$ CHF $116+$ CHF $341+$ $(\mathrm{CHF} 1,920 \times 0.75 \times 0.79)=\mathrm{CHF} 15,734.1+\mathrm{CHF} 6,854.86=\mathrm{CHF}$ $22,588.96=€ 14,570$ (rounded).

\section{Cost-effectiveness}

In the current model, the incremental cost-effectiveness ratios (ICER) of the different strategies were assessed, defined as $\left(\mathrm{C}_{\mathrm{T}}-\mathrm{C}_{\mathrm{N}}\right) /\left(\mathrm{E}_{\mathrm{T}}-\mathrm{E}_{\mathrm{N}}\right)$, where $\mathrm{C}_{\mathrm{T}}-\mathrm{C}_{\mathrm{N}}$ is the difference between the sum of the costs of LTBI treatment $(\mathrm{T})$ minus the costs for no treatment $(\mathrm{N})$ over the 20-yr period, and $\mathrm{E}_{\mathrm{T}}-\mathrm{E}_{\mathrm{N}}$ is the difference between the effectiveness of these so-called "interventions". Effectiveness is measured in terms of the number of cases of TB disease avoided and/or the sum of saved life expectancy (generally converted to "life years gained" (LYG)) to yield the net cost required to increase by one of these additional nonmonetary outcome units compared with the next less costly intervention. Negative numbers thus identify cost savings (if an intervention costs less and is more effective than its comparator) while positive numbers indicate additional expenditure per outcome unit. The higher the ratio, the less cost-effective the intervention.

Quality-adjusted life-years, the effect of interest in most other cost-effectiveness analyses, taking into account both quantity and the quality of life (and therefore affording a weight on time in different health states) have not yet been validated in any depth in connection with $\mathrm{TB}$ and were therefore not included in the present analysis. Future costs and LYG were discounted at an annual rate of $3 \%$.

While the question of what constitutes good value depends on ethical considerations, a rough benchmark of US\$ 50,000 (or $€ 40,195$; average exchange rate for 2004: US $\$ 1=€ 0.8039$ ) per LYG has commonly been used; this is based on Medicare's decision in the 1970s to cover dialysis in patients with chronic renal failure in the USA at a cost-effectiveness ratio within this range [28]. Accordingly, this threshold was used as an indicator of willingness to pay for a healthcare intervention also in Switzerland.

In addition, the total cost for each strategy is presented; broken down by treatment cost, cost due to negative test results and the contribution of costs of overlooked TB cases among false negative contacts with undetected LTBI due to the differing detection sensitivities of each strategy. The average cost-effectiveness, defined as costs per case prevented within a given strategy, is also presented.

\section{Sensitivity analysis}

Sensitivity analyses were performed to examine the impact of uncertainty surrounding the basic model assumptions. Key parameters in this decision analysis model were varied over reasonable ranges to determine the robustness of the costeffectiveness estimate and to determine which parameters were the most important determinants in the model. Variables explored in these analyses included the annual probability of progression to disease following a positive T-SPOT.TB test (with a higher risk modelled, equivalent to the rate of progression following a $15 \mathrm{~mm}$ TST), total cost of TB treatment (with regard to possible future changes in this), and cost of isoniazid (which accounts for $\sim 52 \%$ of the prevention cost and is thus the greatest single cost factor). Thresholds were determined above/below which cost savings could be achieved.

Multivariate sensitivity analyses were performed on the likelihood of progression to active disease, on treatment costs for TB and on cost of isoniazid. The sensitivity and specificity of the TST at the different cut-offs were not changed, because these parameters are directly related to the side-by-side $\mathrm{T}$ SPOT.TB values as conditional probabilities and cannot be evaluated in isolation.

\section{RESULTS}

The projected clinical and economic outcomes of the different screening strategies in the two cohorts are presented in tables 5 and 6 .

\section{A 20-yr-old cohort of close contacts} T-SPOT.TB

On the basis of T-SPOT.TB results, 277 contacts from the hypothetical cohort of 1,000 would be deemed as infected. In the absence of any intervention, a total of 19.6 TB cases would result from these 'test-positives' over 20 yrs. On the basis of screening with T-SPOT.TB and subsequent treatment with isoniazid, 15.6 of these cases could be prevented, saving 10.3 days of life (0.0283 life-yrs) per treated contact and costs of disease amounting to $€ 227,292(15.6 \times € 14,570)$. Assuming a sensitivity of only 0.95 for T-SPOT.TB in the base case, one additional case of TB would have resulted from unrecognised false negative results.

For the high reactivation probability scenario (i.e. assuming the same reactivation probability for T-SPOT.TB as that from a $\geqslant 15 \mathrm{~mm}$ TST result), 29.2 cases would occur, 23.1 of which would be prevented by isoniazid treatment and 1.5 cases would be missed from false negatives The effectiveness of the screening strategies was also calculated on the basis of the number of contacts treated to prevent one active TB case; this was calculated as 18 contacts treated per case prevented $(95 \%$ confidence interval (CI) 11-43) for a normal reactivation probability and 12 (95\% CI 7-59) for a high reactivation probability with the T-SPOT.TB test.

Turning to the costs, under base case assumptions, $€ 104,432$ of a total of $€ 441,310$ (23.7\%) is expended upon negative results comprising the costs of the 723 negative T-SPOT.TB screening tests and $€ 12,836$ upon the consequences of false-negative 
TABLE 5 Health and economic outcomes: 20-yr-old cohort

\begin{tabular}{|c|c|c|c|c|c|}
\hline \multirow[t]{2}{*}{ Model outcomes } & \multicolumn{5}{|c|}{ Screening strategy } \\
\hline & T-SPOT.TB & $\mathrm{TST} \geqslant 5 \mathrm{~mm}$ & $\mathrm{TST} \geqslant 10 \mathrm{~mm}$ & $\mathrm{TST} \geqslant 15 \mathrm{~mm}$ & $\begin{array}{c}\text { TST } \geqslant 10 \mathrm{~mm} / \\
\text { T-SPOT.TB }\end{array}$ \\
\hline Individuals tested positive & 277 & 798 & 723 & 412 & initially 723 \\
\hline Test-positive individuals carrying LTBI & 277 & 273 & 258 & 191 & 258 \\
\hline Active cases averted by treatment & 15.6 & 12.6 & 14.6 & 15.9 & 14.6 \\
\hline Active cases missed from false negatives & 1.0 & 1.1 & 2.4 & 10.7 & 2.4 \\
\hline \multicolumn{6}{|l|}{ Base assumptions } \\
\hline Total cost for 1000 contacts (treated) $€$ & 441310 & 812134 & 762538 & 565873 & 421742 \\
\hline Due to treatment & 336968 & 795740 & 730599 & 438211 & 319030 \\
\hline Due to negative results & 104432 & 16394 & 31939 & 127662 & 102712 \\
\hline from INH treatment days (yrs) & $10.3(0.0283)$ & $2.9(0.00789)$ & $3.7(0.0101)$ & $7.2(0.0196)$ & $10.3(0.0283)$ \\
\hline Number needed to treat to prevent one TB case & $18(11-43)$ & $63(40-158)$ & $50(32-106)$ & $26(19-42)$ & $18(11-43)$ \\
\hline \multicolumn{6}{|l|}{ Thresholds for overall cost savings } \\
\hline If total TB treatment costs increase to $€$ (change) & $22463(+7893)$ & $80445(+65875)$ & $62982(+48412)$ & $32681(+18111)$ & $22463(+7893)$ \\
\hline If INH costs decrease to $€$ (change) & $154(-328)$ & & & & $154(-328)$ \\
\hline \multicolumn{6}{|l|}{ Higher T-SPOT.TB reactivation rate assumption ${ }^{\#}$} \\
\hline $\begin{array}{l}\text { Absolute numbers of TB cases predicted in } \\
\text { absence of intervention }\end{array}$ & 29.2 & & & & \\
\hline Active cases averted by treatment & 23.1 & & & & \\
\hline Active cases missed from false negatives & 1.5 & & & & \\
\hline Total cost for 1000 contacts (treated) $€$ & 468651 & & & & \\
\hline Number needed to treat to prevent one TB case & $12(7-59)$ & & & & \\
\hline \multicolumn{6}{|l|}{ Thresholds for overall cost savings } \\
\hline If total TB treatment costs increase to $€$ (change) & $15112(+542)$ & & & & \\
\hline If INH costs decrease to $€$ (change) & $448(-34)$ & & & & \\
\hline
\end{tabular}

Data are presented as $n$ or $n$ (95\% confidence interval), unless otherwise stated. TST: tuberculin skin test; LTBI: latent tuberculosis (TB) infection; ICER: incremental costeffectiveness ratio; LYG: life-year gained; INH: isoniazid. ${ }^{*}$ : transition probability for a progression to active TB=0.0056.

results $(2.9 \%)$. The incremental cost-effectiveness comparing LTBI treatment versus nontreatment is therefore $€ 11,621 \cdot \mathrm{LYG}^{-1}$, rapidly improving to only $€ 854 \cdot \mathrm{LYG}^{-1}$ when the high reactivation probability is used; the cost per case prevented is $€ 28,289$ and $€ 20,288$, respectively.

Reducing the cost of isoniazid medication in sensitivity analysis to $€ 154$ (a level more comparable with the rest of Europe) would result in an overall cost saving (negative ICER) with T-SPOT.TB when LTBI treatment is offered. This would also be the case if the costs resulting from TB disease were $>€ 22,463$.
Assuming the high reactivation probability, even a very small decrease in isoniazid cost to $€ 448$ (by $\sim 7 \%$ ) would be enough to make a T-SPOT.TB cost saving overall, as would only a modest increase of $3.7 \%$ in the assumed cost of TB disease (from $€ 14,570$ to $€ 15,112$ ).

\section{TST $\geqslant 5 \mathrm{~mm}$}

Performing the TST with a cut-off at $5 \mathrm{~mm}$ resulted in a total of 798 test-positives, 2.88 times as many as obtained by TSPOT.TB, resulting in a large number (525 (798-273)) of contacts assumed to be offered isoniazid unnecessarily. 
TABLE 6 Health and economic outcomes: 40-yr-old cohort

\begin{tabular}{|c|c|c|c|c|c|}
\hline \multirow[t]{2}{*}{ Model outcomes } & \multicolumn{5}{|c|}{ Screening strategy } \\
\hline & T-SPOT.TB & TST $\geqslant 5 \mathrm{~mm}$ & $\mathrm{TST} \geqslant 10 \mathrm{~mm}$ & $\mathrm{TST} \geqslant 15 \mathrm{~mm}$ & $\begin{array}{l}\text { TST } \geqslant 10 \mathrm{~mm} / \\
\text { T-SPOT.TB }\end{array}$ \\
\hline Individuals tested positive & 277 & 798 & 723 & 412 & initially 723 \\
\hline Test-positive individuals carrying LTBI & 277 & 273 & 258 & 191 & 258 \\
\hline Active cases averted by treatment & 11.8 & 9.6 & 11.0 & 12 & 11.0 \\
\hline Active cases missed from false negatives & 0.77 & 0.84 & 1.8 & 8 & 1.8 \\
\hline \multicolumn{6}{|l|}{ Base assumptions } \\
\hline Total cost for 1000 contacts (treated) $€$ & 427792 & 801041 & 746191 & 525889 & 406334 \\
\hline Due to treatment & 326176 & 787437 & 720543 & 426726 & 309870 \\
\hline \multicolumn{6}{|l|}{ Average increased life expectancy per patient } \\
\hline from INH treatment days (yrs) & $7.3(0.0201)$ & $2.1(0.00567)$ & $2.6(0.00718)$ & $5.0(0.0138)$ & $7.3(0.0201)$ \\
\hline Number needed to treat to prevent one TB case & $24(14-75)$ & $83(49-268)$ & $66(41-171)$ & $34(24-61)$ & $24(14-75)$ \\
\hline \multicolumn{6}{|l|}{ Thresholds for overall cost savings } \\
\hline If total TB treatment costs increase to $€$ (change) & 29759 (+15189) & $105401(+90831)$ & $83404(+68834)$ & $43442(+28872)$ & $29759(+15189)$ \\
\hline If INH costs decrease to $€$ (change) & $6.01(-475.99)$ & & & & $6.01(-475.99)$ \\
\hline \multicolumn{6}{|l|}{ Higher T-SРOT.TB reactivation rate assumption ${ }^{\#}$} \\
\hline $\begin{array}{l}\text { Absolute numbers of TB cases predicted in } \\
\text { absence of intervention }\end{array}$ & 21.9 & & & & \\
\hline Active cases averted by treatment & 17.4 & & & & \\
\hline Active cases missed from false negatives & 1.3 & & & & \\
\hline Total cost for 1000 contacts (treated) $€$ & 447852 & & & & \\
\hline Number needed to treat to prevent one TB case & $16(10-36)$ & & & & \\
\hline \multicolumn{6}{|l|}{ Thresholds for overall cost savings } \\
\hline If total TB treatment costs increase to $€$ (change) & $20194(+5624)$ & & & & \\
\hline If INH costs decrease to $€$ (change) & $225(-257)$ & & & & \\
\hline
\end{tabular}

Data are presented as $n$ or $n$ (95\% confidence interval), unless otherwise stated. TST: tuberculin skin test; LTBI: latent tuberculosis (TB) infection; ICER: incremental costeffectiveness ratio; LYG: life-year gained; INH: isoniazid. " ${ }^{\text {: }}$ transition probability for a progression to active $\mathrm{TB}=0.0042$.

Although the combination of low number of test-negative individuals and high NPV (0.907, see above) resulted only in slightly more than one case (1.1) being missed, the treatment costs are more than double (2.4 times) the comparable TSPOT.TB costs. Thus, the ICER is €96,705. $\mathrm{LYG}^{-1}$, more than eight times as high as with the T-SPOT.TB. The only way this screening strategy could be considered cost-effective under the normal willingness-to-pay threshold is if isoniazid medication could be offered without charge. In that case the ICER would fall to $€ 35,707 \cdot \mathrm{LYG}^{-1}$. Cost savings would be achieved only with unrealistically high $\mathrm{TB}$ costs of $\geqslant € 80,445$. A total of 63 contacts $(95 \%$ CI $40-158)$ would have to be treated to prevent one $\mathrm{TB}$ case and the cost per active $\mathrm{TB}$ case prevented would be $€ 64,455$.

TST $\geqslant 10 \mathrm{~mm}$

A cut-off at $10 \mathrm{~mm}$ for the TST does not substantially reduce treatment costs or ICER, as here too the ratio between the false positives (465 (723-258)) to true-positive contacts (258) remains high at 1.8 . Total treatment costs are only $6.1 \%$ lower, but the costs due to false-negative results are more than two times (2.17) as high because of the lower NPV of the TST at a 10-mm compared with a 5-mm cut-off. These false negatives result in 2.4 missed cases. A reduction of the isoniazid price to $€ 171$ or 
an increase in TB cost to $€ 35,600$ (data not shown) would make this strategy cost-effective at the $€ 40,195 \cdot \mathrm{LYG}^{-1}$ threshold, and cost savings are only apparent if the costs of treating TB are $€ 62,982$. A total of 50 contacts (95\% CI 32-106) would have to be treated to prevent one TB case and the cost per active TB case prevented is $€ 52,229$.

\section{$\mathrm{TST} \geqslant 15 \mathrm{~mm}$}

Using a cut-off of $15 \mathrm{~mm}$ clearly reduces the number of TSTpositive individuals (412 versus 723 for the 10-mm cut-off) and further decreases the proportion of unnecessarily treated individuals $((412-191) / 412=53.6 \%$ versus $(723-258) / 723=64.3 \%$ for a cut-off of $10 \mathrm{~mm})$. Due to a higher PPV than for 5- and 10mm cut-offs, and the high tpReact of 0.0056 per year, the ICER for this base case is the only TST screening strategy that falls below the willingness-to-pay threshold with an ICER of $€ 26,451 \cdot \mathrm{LYG}^{-1}$. Nevertheless, the low NPV leads to a high number of missed cases (10.7) and therefore additional costs of $€ 127,662$ due to false-negative results, i.e. nearly one-quarter $(22.6 \%)$ of the total costs. Furthermore, reducing the isoniazid medication price to zero would only diminish the ICER to $€ 1,800 \cdot \mathrm{LYG}^{-1}$. A cost saving can only be achieved if at the same time the TB treatment cost rises to $€ 15,799$; an increase of $\sim 8 \%$. A total of 26 contacts (95\% CI 19-42) would have to be treated to prevent one $\mathrm{TB}$ case and the cost per active $\mathrm{TB}$ case prevented is $€ 35,589$.

\section{TST $\geqslant 10 \mathrm{~mm}$ followed by T-SPOT.TB}

The introduction of screening first by TST with a cut-off at $10 \mathrm{~mm}$, then by the T-SPOT.TB as a confirmation test has no impact on the ICER compared with the T-SPOT.TB alone, but it does falsely reduce the number of treated contacts presumed to be infected by $9.3 \%$ (258 versus 277 ) after pre-selection by the TST. The resulting lower treatment costs ( $€ 17,938$ less) just outweigh the higher costs induced by the higher number of false-negative contacts $(€ 26,416$ versus $€ 12,836$ for the performance of the T-SPOT.TB test alone). However, owing to the lower number of treated contacts and subsequently lower number of cases avoided, the combination slightly increases the cost per case avoided by $\sim € 597$ (2.1\%), leading to a marginally worse average cost-effectiveness than the T-SPOT.TB alone. The number of contacts needed to treat to avoid one future case of TB is unchanged at 18 (95\% CI 11-43).

\section{0-yr-old cohort of contacts}

The risk of disease in those who were infected is lower in elderly LTBI patients, and therefore the sum of the future cost of TB will be relatively low in comparison with those for the 20-yr-old contacts, because of the lower number of cases of active TB disease. As expenditures for LTBI treatment remain constant, the ICER will (in contrast to the 20-yr-old group) rise rapidly in all strategies applied to the 40-yr-old contacts. Only the T-SPOT.TB-based treatment under basecase estimates, and even more under the high-progression probability assumption, is cost-effective, achieving an ICER of $€ 23,692 \cdot \mathrm{LYG}^{-1}$ and $€ 8,642 \cdot \mathrm{LYG}^{-1}$, respectively. Cost savings can be achieved if the INH costs decline to $€ 6$ and $€ 225$ per treatment course under base case and the high progression probability assumptions, respectively. None of the TST strategies without combination with the T-SPOT.TB are cost-effective under any reasonable combination of other parameters.

\section{DISCUSSION}

Until recently, cost-effectiveness analyses of LTBI treatment were based on outdated assumptions regarding sensitivity and specificity derived from TST parameters. Mostly varying between 95 and $99 \%[16,24,25]$, these could not take into account the results of new scientific discoveries showing the lack of specificity of the TST, and may for this reason lead to a systematic bias by overestimating the number of contacts potentially infected and, therefore, the number of cases prevented as the numerator of the incremental cost-effectiveness ratio. The present authors set out to assess the consequences for cost-effectiveness of screening and treating LTBI patients in Switzerland on the basis of current "real-

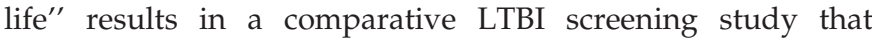
compared the new T-SPOT.TB assay with TST-based strategies among close contacts of infectious pulmonary TB source cases. An inherent limitation of this, and indeed any, analysis designed to compare cost-effectiveness against an imperfect standard (such as the TST) is that there is not a gold-standard test to consult in order to separate discordant results. Various assumptions have to be made as to which test result is more likely to be the correct one as the basis of generating quantitative comparative performance measures. This limitation should be recognised in interpreting the present results.

Although it is not imperative that the implementation of a programme for preventing infectious diseases result in monetary savings to be cost-effective, it cannot be assumed that societies are willing to pay any price for preventive interventions. Therefore any new intervention must have an acceptable cost associated with the health benefits it brings.

In the 20-yr-old close contacts, the baseline strategy of screening combining the TST at a cut-off of $10 \mathrm{~mm}$ and subsequently the T-SPOT.TB was the least costly alternative; however, the most cost-effective on average was the use of TSPOT.TB alone. Referred to the threshold of US\$ 50,000 $(€ 40,195)$ per LYG, no TST-based programmes were costeffective; with the exception of using a $15-\mathrm{mm}$ cut-off in the younger group where the cost-effectiveness (at $€ 26,451 \cdot \mathrm{LYG}^{-1}$ ) fell below the willingness-to-pay threshold. However, this came at the price of producing the highest total cost due to low sensitivity and therefore an unacceptably high rate of missed M. tuberculosis-infected contacts developing TB disease in the future. Using the T-SPOT.TB test, either alone or in combination with the TST, greatly reduced the number of people it was necessary to treat in order to prevent one TB case (from 50 to 18) versus the status quo of TST cut-off $\geqslant 10 \mathrm{~mm}$.

The sensitivity analysis showed that the cost of the isoniazid medication for the 9-month course (currently $€ 482$ in Switzerland) appeared to be the most important cost parameter. For example, if the cost of isoniazid was assumed to be reduced by two-thirds then the two T-SPOT.TB-based strategies become cost-saving; that is, saving both total costs and life-yrs. This is important for the generalisation of the results to other countries as the cost of isoniazid appears to be much 
higher in Switzerland than elsewhere (for example, the cost of 9 months of isoniazid in Germany is $€ 70.20$ [13]), particularly where generic drugs are used.

The risk of progression to active disease after LTBI in the 40-yrold cohort of contact individuals appeared to have the greatest influence on the cost-effectiveness outcome. While reasonably reducing the cost of isoniazid medication would not result in considerable changes in the cost-effectiveness owing to the comparatively low annual reactivation base-case probabilities inherent in older infected individuals, the high-progression assumption led to a low ICER for using the T-SPOT.TB and, combined with a moderate isoniazid price decrease in the sensitivity analysis, even to a cost reduction. This has important implications when the applicability of these findings to the screening of groups at particularly high rates of reactivation, such as HIV-infected patients in both low- and high-prevalence settings, is considered.

Given the importance of the assumed rate of progression to active $\mathrm{TB}$ as a variable in the model, it deserves further discussion. In particular, as there are as yet no long-term prospective follow-up studies showing the risk of developing active TB following a positive blood test (except for one small study [29]), the reactivation probability for T-SPOT.TB-positive individuals is still unknown and this limits the accuracy of this analysis. In the absence of any other data, the present authors assumed that this value for T-SPOT.TB was comparable to that for the TST, using values from a recent meta-analysis [22]. However, this assumption is likely to underestimate the true cost-effectiveness of T-SPOT.TB as its greater sensitivity and specificity should result in a higher PPV than found with the TST. This is because in prospective studies with the TST where the reactivation rate is calculated as from the incidence of active TB disease deriving from a certain number of TSTpositive individuals, a proportion of the followed-up TSTpositives will never have been TB infected owing to the known false-positive results induced by both prior BCG vaccination and nontuberculous mycobacterial infection. This systematic error serves to underestimate the true risk of reactivation in those who were genuinely infected. At the same time, the TST is known to suffer from false-negative results, and these occur disproportionately in those with weaker immune systems. These people are ironically also those who are at greatest risk of reactivation. By excluding these truly infected individuals who were negative to the TST from the subsequent follow-up, the true reactivation rate of those truly infected is again underestimated. Using a test that has higher sensitivity (identifying more of those are at high risk of reactivation) and higher specificity (not identifying uninfected patients) than the TST, the subjects found to be positive can thus be reasonably assumed to have a higher reactivation rate than the TST. If T-SPOT.TB does indeed demonstrate a better PPV for the subsequent development of TB disease than is currently observed with the TST, then the cost-effectiveness of TSPOT.TB-based screening will be dramatically increased from that modelled here. This is an important area for future study.

The possible benefits of the T-SPOT.TB assay are also underestimated owing to the fact that the present model did not include wider transmission of $\mathrm{TB}$ into the community (i.e. the active TB cases that occur themselves infecting new contacts) over the 20 yrs. Adding these to the decision tree would certainly increase the benefits from isoniazid treatment, but it would also make this model even more complex.

Despite these limitations, the current authors believe this study has four important outcomes. First, it illustrates that the historical solely TST-based screening strategies and preventive treatment of LTBI are arguably not cost-effective medical interventions when set against a benchmark of $€ 40,195 \cdot \mathrm{LYG}^{-1}$. Secondly, the current findings show that using T-SPOT.TBbased screening is cost-effective (taking the same measure) in an absolute sense and will be net cost-saving if isoniazid costs are close to international norms. Thirdly, T-SPOT.TB-based screening strategies are significantly cost-saving when compared to the status quo of TST-based TB control programmes. Fourthly, the use of T-SPOT.TB (either alone or in combination with the TST) greatly reduces the number of contacts treated to prevent one TB case, from 26-63 (depending on the cut-off for positive TST) to 18 .

Reducing the number of individuals needing to be treated to avoid one case of TB by a better selection of infected contacts may have important implications in countries with a low incidence of TB as an addition to the global elimination strategies. In high-prevalence countries, particularly in regions where the rate of LTBI among HIV-positive patients is elevated, and considering the fact that the T-SPOT.TB test appears more sensitive and more specific than TST in advanced immunosuppression, such a strategy could also be considered as a possible way to reduce the burden of disease and the costs associated with reactivation of TB by offering preventive treatment to infected patients [30-35].

These findings have important ramifications for healthcare providers in setting new guidelines for the use of this new test, and underline the validity of the new Swiss screening recommendations.

\section{REFERENCES}

1 Broekmans JF, Migliori GB, Rieder HL, et al. European framework for tuberculosis control and elimination in countries with a low incidence. Recommendations of the World Health Organization (WHO), International Union Against Tuberculosis and Lung Disease (IUATLD) and Royal Netherlands Tuberculosis Association (KNCV) Working Group. Eur Respir J 2002; 19: 765-775.

2 Ewer K, Deeks J, Alvarez L, et al. Comparison of T-cellbased assay with tuberculin skin test for diagnosis of Mycobacterium tuberculosis infection in a school tuberculosis outbreak. Lancet 2003; 361: 1168-1173.

3 Zellweger JP, Zellweger A, Ansermet S, de Senarclens B, Wrighton-Smith P. Contact tracing using a new T-cellbased test: better correlation with tuberculosis exposure than the tuberculin skin test. Int J Tuberc Lung Dis 2005; 9: 1242-1247.

4 Diel R, Ernst M, Döscher G, et al. Avoiding the effect of BCG vaccination in detecting Mycobacterium tuberculosis infection with a blood test. Eur Respir J 2006; 28: 16-23.

5 Shams H, Weis S, Klucar P, et al. Enzyme-linked immunospot and tuberculin skin testing to detect latent tubercu- 
losis infection. Am J Respir Crit Care Med 2005; 172: 1161-1168.

6 Richeldi L, Ewer K, Losi M, et al. T cell-based tracking of multidrug resistant tuberculosis infection after brief exposure. Am J Respir Crit Care Med 2004; 170: 288-295.

7 Piana F, Codecasa LR, Cavallerio P, et al. Use of a Tcell-based test for detection of tuberculosis infection among immunocompromised patients. Eur Respir J 2006; 28: $31-34$.

8 Diel R, Nienhaus A, Lange C, Schaberg T. Cost-optimisation of screening for latent tuberculosis in close contacts. Eur Respir J 2006; 28: 35-44.

9 Wrighton-Smith P, Zellweger J-P. Direct costs of three models for the screening of latent tuberculosis infection. Eur Respir J 2006; 28: 45-50.

10 Swiss Lung League. Erkennung der Tuberkuloseinfektion mittels Bluttest (Interferon-gamma). [Detection of tuberculous infection by a blood-test (interferon-gamma).] Bern, Lungenliga Schweiz, October 2005 (updated December 19, 2005).

11 Lee JY, Choi HJ, Park I-N, et al. Comparison of two commercial interferon- $\gamma$ assays for diagnosing Mycobacterium tuberculosis infection. Eur Respir J 2006; 28: 24-30.

12 Lalvani A, Pathan A, McShane H, et al. Rapid detection of Mycobacterium tuberculosis infection by enumeration of antigen-specific T cells. Am J Respir Crit Care Med 2001; 163: 824-828.

13 Ferrara G, Losi M, D'Amico R, et al. Use in routine clinical practice of two commercial blood tests for diagnosis of infection with Mycobacterium tuberculosis: a prospective study. Lancet 2006; 367: 1328-1334.

14 Diel R, Nienhaus A, Schaberg T. Cost-effectiveness of isoniazid chemoprevention in close contacts. Eur Respir J 2005; 26: 465-473.

15 Chapman A, Munkanta M, Wilkinson K, et al. Rapid detection of active and latent tuberculosis infection in HIVpositive individuals by enumeration of Mycobacterium tuberculosis-specific T cells. AIDS 2002; 16: 2285-2293.

16 Pathan AA, Wilkinson KA, Klenerman $\mathrm{P}$, et al. Direct ex vivo analysis of antigen-specific IFN- $\gamma$-secreting CD4-T cells in Mycobacterium tuberculosis-infected individuals; associations with clinical disease status and effect of treatment. J Immunol 2001; 167: 5217-5255.

17 Lalvani A, Nagvenkar P, Udwadia Z, et al. Enumeration of $\mathrm{T}$ cells specific for RD1-encoded antigens suggests a high prevalence of latent Mycobacterium tuberculosis infection in healthy urban Indians. J Infectious Dis 2001; 183: 469-477.

18 Meier T, Eulenbruch HP, Wrighton-Smith P, Enders G, Regnath T. Sensitivity of a new enzyme-linked immunospot assay (T SPOT-TB) for diagnosis of tuberclosis in clinical practice. Eur J Microbiol Infect Dis 2005; 24: 529-536.
19 Swiss Federal Statistical Office. Sterbetafeln für die Schweiz 1998/2003. [Mortality Tables for the Swiss 1998/ 2003.] Bern, Swiss Federal Statistical Office, 2005.

20 Swiss Federal Statistical Office. Individual assessment.

21 Horsburgh CR Jr. Priorities for the treatment of latent tuberculosis infection in the United States. $N$ Engl J Med 2004; 350: 2060-2079.

22 Swiss Lung League-Swiss Federal Statistical Office. Handbuch Tuberkulose. [Tuberculosis Handbook]. Schweiz Med Forum 2003; 3: 487-491.

23 Swiss Federal Office of Public Health. Bulletin 20/06. Communicable Diseases. Tuberculosis in the Swiss 20012004. Bern, Swiss Federal Office of Public Health, 2006; p. 428.

24 Nicolet G, Rochat T, Zellweger JP. Traitement de la tuberculose. [Treatment of tuberculosis.] Swiss Medical Forum 2003; 22: 506-516.

25 Hanoverian consensus group. German revised recommendations on health economic evaluation. Deutsch Med Wochenschr 1999; 124: 1503-1506.

26 Swiss Federal Statistical Office. Löhne, Erwerbseinkommen - Indikatoren. [Salaries and earnings: indicators.] www.bfs.admin.ch/bfs/portal/de/index/themen/03/04/ blank/key/erwerbseinkommen.html. Date last updated: May 8, 2007. Date last accessed: May 8, 2007.

27 Swiss Federal Statistical Office. Taschenstatistik der Schweiz, Arbeit. [Statistical data on Switzerland 2006. Work.] Bern, Swiss Federal Statistical Office, 2006; p. 8.

28 Owens DK. Interpretation of cost-effectiveness analyses. J Gen Intern Med 1998; 13: 716-717.

29 Doherty TM, Demissie A, Olobo J, et al. Immune responses to the Mycobacterium tuberculosis-specific antigen ESAT-6 signal subclinical infection among contacts of tuberculosis patients. J Clin Microbiol 2002; 40: 704-706.

30 Fallab-Stubi CL, Zellweger JP, Sauty A, Uldry C, Iorillo D, Burnier M. Electronic monitoring of adherence to treatment in the preventive chemotherapy of tuberculosis. Int $J$ Tuberc Lung Dis 1998; 2: 525-530.

31 Nunn P, Williams B, Floyd K, Dye C, Elzinga G, Raviglione M. Tuberculosis control in the era of HIV. Nat Rev Immunol 2005; 5: 819-826.

32 Harries AD, Boxshall M, Phiri S, Kwanjana J. Managing HIV and tuberculosis in sub-Saharan Africa. Lancet 2006; 367: 1817-1818.

33 Woldehanna S, Volmink J. Treatment of latent tuberculosis infection in HIV infected persons. Cochrane Database Syst Rev 2004; 1: CD000171.

34 Shrestha RK, Mugisha B, Bunnell R, et al. Cost-effectiveness of including tuberculin skin testing in an IPT program for HIV-infected persons in Uganda. Int J Tuberc Lung Dis 2006; 10: 656-662.

35 Mugisha B, Bock N, Mermin J, et al. Tuberculosis case finding and preventive therapy in an HIV voluntary counseling and testing center in Uganda. Int $J$ Tuberc Lung Dis 2006; 10: 761-767. 